\title{
The Digitalization of Chinese Paper-Cut: Intangible Cultural Heritage Protection and Culture Communication
}

\author{
Junxi Chen ${ }^{1, a}$, Desheng Lyu ${ }^{2, b^{*}}$ \\ ${ }^{1}$ Harbin Institute of Technology, 92 West Dazhi Street, Harbin 150001, China \\ ${ }^{2}$ Harbin Institute of Technology, 92 West Dazhi Street, Harbin 150001, China \\ acjx7seven@163.com , bdeshengl@hit.edu.cn
}

Keywords: Intangible cultural heritage;Chinese paper-cut;Digital thchnique; Culture communication; Culture protection

\begin{abstract}
As an important wealth of humanity, the intangible cultural heritage digital protection has been done a lot of work. The research on digital protection of China started relatively late. How to use digital means of effect protection and propagation chinese intangible cultural heritage has been imminent. China's paper cutting, is the unique artistic treasures of the Chinese nation. It is an important representative of Chinese intangible cultural heritage and has been listed in the world intangible cultural heritage protection list. For the purpose of study Chinese intangible cultural heritage digital protection and culture communication, an case study of digital Chinese paper-cutting art was accomplished. The history and current situation of the Chinese paper-cut art was introduced. At the same time analyze and discuss the experience and application of the virtual actting in order to show digital means' effect and significance in chinese intangible cultural heritage protection and propagation. Finally, An analysis of how paper-cut culture spread was put forward.
\end{abstract}

\section{Introduction}

In the present era, "intangible cultural heritage", a household term, has become synonymous with culture. Intangible cultural heritage is precious human treasure, closely related to the inheriting of the historical civilization and spiritual civilization of a country or a nation. Different from tangible heritage, intangible cultural heritage is like sand on the fingertips. Without careful protection, it can be lost easily. Once lost, it can be irrevocable. In the face of the current situation of the intangible cultural heritage gradually disappearing, protection of intangible cultural heritage is becoming more widely concerned by governments and academia of all countries in the world. United Nations Educational, Scientific and Cultural Organization has made many efforts in digital protection of non-material cultural heritage. The research of the method of digital protection for cultural heritage was formally included in the plan by the UNESCO in 2002[1]. In such circumstances, developing effective means of digital protection becomes very important in protecting and inheriting China's intangible cultural heritage and it is also our responsibility.

\section{The History and Current Situation of the Development of Paper-cutting}

The History of Paper-cutting. China's paper cutting, is the unique artistic treasures of the Chinese nation, an outstanding folk art of China, can bring us spiritual pleasure and visual enjoyment[2].

According to archaeological speculation, the art of paper-cutting was originally discovered in the Northern Dynasty, dating back more than one thousand five hundred years ago. The original paper-cutting should appear in the Han Dynasty. The invention of paper played an important role in the appearance, development and popularization of paper-cutting. Gradually, the art of paper-cutting experienced a period of great development of the Tang Dynasty. The maturity of the paper-making technology in the Song Dynasty provided the conditions for the popularity of paper cutting, which, then, became mature in the Ming and Qing Dynasty, reaching its best time. From China's five 
thousand years of cultural accumulation originated the essence of traditional Chinese culture. Papercutting, as a part of Chinese traditional folk arts, has a long history and enjoys long-term popularity.

In China, the art of paper-cutting is in different forms, whether in technique or styles, whether in the south or in the north. Different kinds of paper-cutting reflect different customs in different places. But generally speaking, paper-cutting can be divided into paper-engraving, flower-cutting and window decorations in terms of production forms. And the tools used in paper-cutting are usually scissors and gravers ect(refer with: Fig. 1 Paper-cutting tools). The material used in paper-cutting includes paper, gold or silver leaf, cloth, and animal skins etc.

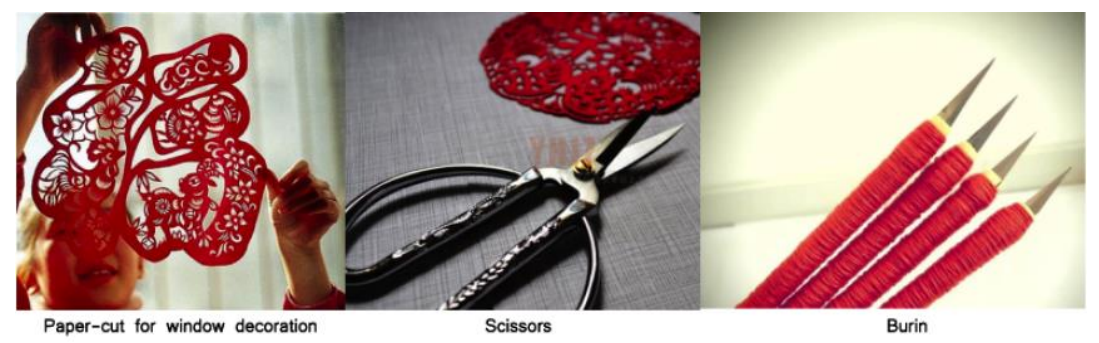

Figure. 1

The Current Situation of the Development of Paper-cutting Art. The art of Chinese paper-cutting is a good example of human being's intangible cultural heritage. The specialized departments for Chinese paper-cutting and the folk protection organizations for it both intensify efforts to promote the protection and spread of the art of paper-cutting. The UNESCO also gives high evaluation on the artistic value of paper-cutting.

Originating from embroidery, the most common Chinese paper-cutting are window decorations. It has become a social custom nowadays, since many Chinese people are delighted to put on some window decorations whenever they celebrate a festival. What they do actually reflects their expectation for a wonderful life in future. Although paper-cutting is well-known all over the world, it is becoming more or less ignored, which makes it difficult in developing and inheriting paper-cutting as a Chinese traditional art. In recent years, Chinese governments of all levels and folk paper-cutting artists have begun to pay more attention to the exploration, protection and inheritance of paper-cutting. Take paper-cutting in Guangling County, Shanxi Province for example, Zhang Duotang, a descendant of paper-cutting in Guangling County, established the first museum of paper-cutting in China, which is called Guangling Paper-cutting Museum. It is also the only industrial district of the national intangible cultural heritage in form of paper-cutting. Meanwhile, in order to sovle the problem of lacking experts, Guangling County opens training schools for paper-cutting to encourage more youths to devote themselves to the cause of paper-cutting, and makes every effort to promoting its development. What Guangling people have done to this form of art helps to establish a platform for Guangling paper-cutting to step into the world.

In the very beginning, there is no difference between "folk" and "official" art of paper-cutting. However, paper-cutting tends to be popular in common people's life with the development of economy and the change of time. Therefore, the themes of paper-cutting works are familiar to the common people. At present, it is difficult for us to see some traditional paper-cutting works, such as Menzhan, window decorations, and hi flowers whether in the rural areas or metropolises. With time going by, people gradually leave paper-cutting behind, making it a dying skill. For instance, we now can hardly see paper-cutting decorations in cave-houses of North Shanxi, which used to be very common in the past. We are supposed to protect and spread the paper-cutting art in a digital way, so that it can have a brand new future. 


\section{The Strategies of Digital Protection and Spread of Paper-cutting}

Paper-cutting Experience Based on "Interactive Virtual Reality Technology". With the improvement of interactive virtual reality technology, people become more and more interested in the sense of reality and amusement the interactive virtual reality technology brings us. Nowadays, it becomes essential to meet people's personal requirements, follow the trend of science development, and explore the digital ways of paper-cutting protection.

Now, interactive virtual reality technology has been widely applied in all kinds of areas. For example, the famous digital Michelangelo project by Leland Stanford Junior University, University of Washington and Cyberware corporation[3]; The virtual cultural relic VIHA 3D project by EU[4]; The Rome Grand Theater digital project by Sapienza University of Rome, University of Ferrara and University of California-Berkeley[5]; The Sulman mummy project by University of Chicago and University of Western Ontario[6]; The digital protection for the lion dance- the living cultural heritage in Ozzy area of Japan by Ozzy University[7]; The virtual Wuqinxi interactive system by Beijing University of Aeronautics \& Astronautics[8]. In view of the application examples of virtual reality interactive technology, the users can finish the process of paper-cutting in reality using interactive virtual reality technology if we apply it to paper-cutting and make it a virtual paper-cutting interactive system(refer with: Fig. 2 a.The virtual paper-cutting interactive system). In this system, The users can use virtual operating system tools to create virtual paper-cutting works when the real operating system tools are replaced by the virtual ones and the real paper is replaced by the virtual one as well. It's easy and completely possible to use virtual paper-cutting interactive system to avoid the complication of the real paper-cutting. At the same time, virtual paper-cutting interactive system is the combination of technology and art. It is a good way to promote the protection and spread of the art of Chinese paper-cutting in digital ways.

Discussion on the Application of Virtual Paper-cutting Interactive System. Application in classes. Nowadays, paper-cutting is usually taught only by appreciating the art of paper-cutting, which makes it impossible for students to learn this art quite well without real practice. If the virtual paper-cutting interactive system is widely applied in classes, students will experience the process of paper-cutting. They can not only feel the pleasure brought by virtual paper-cutting, but also develop more interest in it. It will surely promote and develop the art of paper-cutting inheritance when students have a good knowledge of paper-cutting and then become the heirs of it(refer with: Fig. 2 b.Application in classes).

Application in paper-cutting art museums. Today, paper-cutting art museums are being built in many places in China. which are mainly aimed at the introductions of the culture and history about paper-cutting. Meanwhile, some paper-cutting art works are being exhibited in the museums where the visitors can hardly experience the process of paper-cutting by themselves. If the virtual paper-cutting interactive system can be applied in the paper-cutting museums, it will bring pleasure to the visitors, enable more and more people to learn the culture of paper-cutting and attract more people's attention and interest. It will carry forward the excellent national culture, create a Chinese characteristic culture brand, and offers a cultural atmosphere where classics are inherited, visitors all enjoy themselves, and the good works are shared together. The art of paper -cutting will certainly attract the attention of people all of the world. Our Chinese people will make friends with those who love paper-cutting. We can also do business related to paper-cutting, which will in turn promote the development of the tourism(refer with: Fig. 2 c.Application in paper-cutting art museums). 


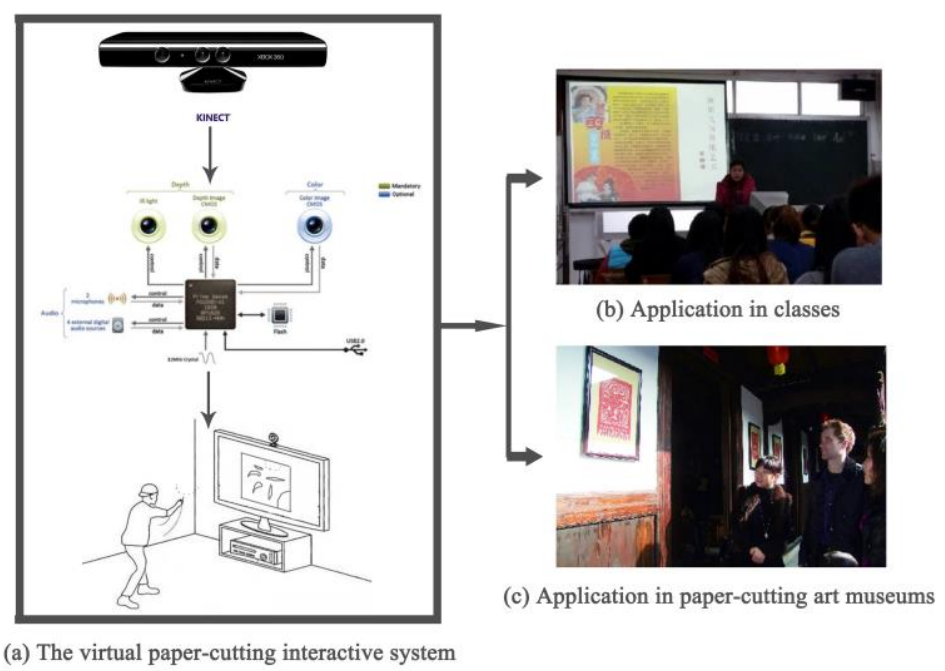

Figure. 2

The virtual paper-cutting interactive system can be applied in many areas. As a digital means of protection for paper-cutting art, the virtual paper-cutting interactive system, with the real purpose of protecting and spreading the art of paper-cutting, can achieve its purpose only by being applied to proper areas.

\section{Summary}

The art of paper-cutting shows the wisdom of Chinese ancestors, who created lots of long-lasting works. The art of Chinese paper-cutting is a mother culture, and also our roots. It flows in our body and offers us emotions. However, it is dying out quickly. So we must strengthen the digital protection and inheritance of it, and try to spread it to the whole world. With the help of the digital technique to protect and carry forward the intangible cultural heritage, it has been an inevitable trend $[9,10]$. Discussion on paper-cutting interactive system is a creative way of protecting the art of paper-cutting and at the same time it can point out the right direction for the digital protection and spread of Chinese intangible cultural heritage.

\section{Acknowledgements}

This research is funded by Heilongjiang Provincial Natural Science Funds of China (Grant NO.: LC2013C19).

\section{References}

[1] $\mathrm{Gu}$ ben, Guidelines on the digital protection for cultural heritage and the UNESCO[J]. Journal of the National Library, 2003(1)

[2] Xiao bin, Analysis of the current development Chinese paper-cut art[J]. He Bei Tourism Professional College, 2008. 03: 144-146.

[3] Marc L Evoy, The digital michelangelo project[C]. Second International Conference on 3D Imaging and Modeling, (1999)

[4] P Cignoni, Adaptive TetraPuzzles: Efficient-out-of core construction and visualization of gigantic multiresolution polygonal models[J]. ACM Transaction Graphics, 2004. 23(3): 796-803. 
[5] Alonzo C Addison, Emerging trends in virtual heritage[J]. IEEE Multi-Media. 2007, 7(2):

222-225.

[6] Janet C Gardner, Greg Garvin, Paleoradiology in mummy studies: the Sulman Mummy Project, Canadian Association of Radiologists Journal. Oct 2004. 55(4): 228-234.

[7] William L, Martens, Digital Abstraction of Sound and Motion for Virtual Preservation of Living Cultural Heritage: The "Chain of Spirits". Proc. 9th Int. Conf. on Virtual Systems and Multimedia, Montreal, QC, Oct. 15. 2003: 76-82.

[8] YUE QI, XU2KUN SHEN, QIN2PING ZHAO, Virtual five animals exercise[C]. Edutainment 2006, LNCS 2006: 39-42.

[9] Fei long, The present situation of the abroad to protect intangible cultural heritage[J]. Theory and Criticism of Literature and Art, 2005(6)

[10] Diana M. Zorich, A Survey of Digital Culture Heritage Initiatives and Their Sustainability[R]. Council on Library and Information Resource(CLIR), Washingto D.C. (2003) 